\title{
Interprétation des données physico-chimiques et biocinétiques pour le calcul de dose : exemple d'un composé industriel $\mathrm{UO}_{2}$ appauvri fabriqué pour le combustible MOX
}

\author{
E. ANSOBORLO*, V. CHAZEL, P. HOUPERT, \\ M.H. HENGÉ-NAPOLI, F. PAQUET
}

(Manuscrit reçu le 17 mars 1997, accepté le 7 juin 1997)

RÉSUMÉ La Commission Internationale de Protection Radiologique (CIPR), dans ses nouvelles recommandations, propose l'utilisation de paramètres déterminés expérimentalement pour effectuer un calcul de dose efficace par unité d'incorporation (DPUI) spécifique pour chaque composé. Le but de cette étude est de définir une méthodologie (techniques d'analyse, test in vitro de dissolution et test in vivo sur rats) permettant d'accéder à ces données et de présenter les méthodes de calcul utilisées, comme le logiciel GIGAFIT pour l'interprétation des données de transfert sanguin, et le logiciel LUDEP pour le calcul de dose. Ce travail a permis de déterminer pour un composé $v O_{2}$ (uranium appauvri), utilisé dans la fabrication du combustible MOX, les principaux paramètres physico-chimiques et biologiques qui entrent dans le calcul de dose : le Diamètre Aérodynamique Médian en Activité ou DAMA de $6,5 \mu \mathrm{m}$; la Surface Spécifique $S S=2,68 \mathrm{~m}^{2} \mathrm{~g}^{-1}$; et les fraction et taux de transfert sanguin respectivement $f_{\mathrm{r}}$, entre $2,5 \%$ et $44,4 \%, s_{\mathrm{r}}$, entre 0,09 et $1,7 \mathrm{j}^{-1}$, et $s_{\mathrm{S}}$, entre $6,4 \times 10^{-4}$ et $1,5 \times 10^{-3} \mathrm{j}^{-1}$. Ces résultats intégrés dans LUDEP conduisent à une DPUI spécifique par inhalation pour ce composé UO égale à $2,84 \times 10^{-6} \mathrm{~Sv} \mathrm{~Bq}^{-1}$.

ABSTRACT Interpretation of physico-chemical and biokinetic data for dose calculation: Example of an industrial depleted $\mathrm{UO}_{2}$, used in the MOX fuel manufacture. The implementation of the new recommendations of the International Commission on Radiological Protection (ICRP) allows to use experimental parameters in order to calculate a specific effective Dose Per Unit of Intake (DPUI) for each compound. The aim of this study is to define a methodology (including analysis techniques, in vitro dissolution tests and in vivo experiment in rats) leading to the determination of these parameters, and to present different calculation methods such as GIGAFIT software for the interpretation of blood translocation data, and LUDEP software for the assessment of effective dose. This study has led, for an industrial uranium oxide compound $\mathrm{UO}_{2}$ (depleted uranium), which is used in the MOX fuel manufacture, to the assessment of the main physico-chemical and biological parameters entering into dose calculation: the average Activity Median Aerodynamic Diameter

\footnotetext{
* Institut de Protection et de Sûreté Nucléaire, département de protection de la santé de l'homme et de dosimétrie, service de dosimétrie, IPSN, BP $n^{\circ} 38,26701$ Pierrelatte cedex, France.
} 


\begin{abstract}
AMAD $=6.5 \mu \mathrm{m}$; the specific surface $S S=2.68 \mathrm{~m}^{2} \mathrm{~g}^{-1}$; and the fraction and rate of transfer to blood which are ranging from $2.5 \%$ to $44.4 \%$ for $f_{\mathrm{r}}, 0.09$ to $1.7 \mathrm{~d}^{-1}$ for $s_{\mathrm{r}}$ and $6.4 \times 10^{-4}$ to $1.5 \times 10^{-3} \mathrm{~d}^{-1}$ for $s_{\mathrm{s}}$. All these results were inserted in LUDEP to determine the specific inhalation DPUI value given for this industrial $\mathrm{UO}_{2}$ as equal to $2.84 \times 10^{-6} \mathrm{~Sv} \mathrm{~Bq}^{-1}$.
\end{abstract}

\title{
1. Introduction
}

La mise en place des nouvelles recommandations de la Commission Internationale de Protection Radiologique dans la publication 60 (ICRP, 1990), du nouveau modèle dosimétrique des voies respiratoires dans la publication 66 (ICRP, 1994), et la parution de la nouvelle Directive européenne (Journal Officiel, 1996) ont permis de modifier l'approche de la dosimétrie interne liée à la surveillance des travailleurs. Ces recommandations ont conduit, dans le domaine du calcul de dose, à une terminologie de référence qui est la Dose efficace Par Unité d'Incorporation ou DPUI. La différence essentielle avec les anciennes recommandations de la publication 30 (ICRP, 1979), et la législation française encore en vigueur, est la flexibilité du calcul de dose. Celui-ci est soit basé sur un ensemble de valeurs par défaut, soit sur la détermination expérimentale d'un certain nombre de paramètres spécifiques conduisant à l'évaluation d'une DPUI propre à chaque composé.

On peut distinguer plusieurs types de paramètres dont certains sont mesurables expérimentalement de façon simple et directe. Il s'agit du Diamètre Aérodynamique Médian en Activité (DAMA) et de l'écart type géométrique $\sigma_{\mathrm{g}}$. Lorsque que le DAMA n'est pas mesuré, un paramètre tel que la densité permet de calculer ce DAMA à partir de la mesure du diamètre géométrique simple. D'autres paramètres, tels que la Surface Spécifique $(S S)$, ou des facteurs plus difficilement mesurables comme le taux d'hydratation, le vieillissement ou la mouillabilité, ne sont pas directement pris en compte par les modèles. Cependant leur influence sur le calcul de dose peut être estimée au travers des paramètres biologiques de dissolution et de transfert sanguin. Ces derniers, quant à eux, constituent la troisième classe de facteurs entrant dans le calcul de dose. Ces paramètres biologiques sont issus de 2 approches complémentaires. La première consiste en une étude de dissolution in vitro permettant d'avoir une première estimation des valeurs spécifiques d'absorption. La deuxième est une confirmation par des essais sur animaux (inhalation ou instillation) permettant d'affiner les valeurs de transfert sanguin. Ces deux approches nécessitent un travail important de traitement et d'interprétation des données : ceci est réalisé à l'aide du logiciel évolutif GIGAFIT développé par 
le NRPB ${ }^{(1)}$, et les résultats obtenus (paramètres d'absorption au sang) sont ensuite utilisés pour le calcul de dose, réalisé par l'intermédiaire du logiciel LUDEP (Birchall et al., 1991; Jarvis et Birchall, 1994).

Afin d'illustrer ce propos, un exemple d'interprétation des données expérimentales est présenté. Il s'agit d'une étude complète effectuée dans un atelier du cycle du combustible dans lequel est fabriqué le composé $\mathrm{UO}_{2}$ sous forme de poudre. Celui-ci est destiné à être mélangé à des oxydes de plutonium pour former des composés de type MOX. De nombreuses études ont été publiées sur différents composés $\mathrm{UO}_{2}$ industriels. Ces études traitaient suivant les cas : la spéciation des aérosols (Ansoborlo et al., 1995a; Ansoborlo et al., 1995b; Surya Narayana et al., 1994) ; les expériences sur le comportement biocinétique in vitro (Cooke et Holt, 1974; Kalkwarf, 1983) ou in vivo (Ansoborlo et al., 1989; Ansoborlo et al. 1995a ; Eidson, 1994; Métivier et al., 1992 ; Stradling et al., 1988); et enfin la surveillance des travailleurs exposés (Ansoborlo, 1989; Schieferdecker et al., 1985). Ce rapport souligne l'importance de l'utilisation des paramètres expérimentaux spécifiques sur le calcul de dose, sur la prédiction de l'excrétion ou de la rétention dans certains organes, ainsi que sur la comparaison avec les données existantes dans la littérature pour ce composé $\mathrm{UO}_{2}$.

\section{Matériels et méthodes}

Les techniques d'analyse ou d'interprétation utilisées pour la détermination des paramètres spécifiques de spéciation des composés industriels peuvent être divisées en 2 parties:

1/ Les techniques expérimentales conduisant à des déterminations directes de paramètres utilisables à l'état brut pour le calcul de dose et qui ont été décrites dans des publications précédentes (Ansoborlo et al., 1989; Ansoborlo et al., 1995a; Ansoborlo et al., 1995b). Il s'agit, tout d'abord des techniques de caractérisation physico-chimique. Celles-ci incluent les prélèvements atmosphériques et la mesure de la distribution granulométrique (conduisant au DAMA et $\sigma_{\mathrm{g}}$, et les techniques d'évaluation de certains paramètres comme la densité, la surface spécifique, les formes cristallines (Rayons X), et la morphologie (diamètre géométrique en utilisant la Microscopie Électronique à Balayage). Viennent ensuite les études de comportement biocinétique qui comprennent les tests in vitro (dissolution chimique) et les essais in vivo (inhalation ou instillation). Ceux-ci fournissent des données sur la rétention du composé dans les principaux organes cibles (poumon, os et rein dans le cas de l'uranium) et sur l'excrétion au niveau des selles et des urines.

(1) NRPB : National Radiological Protection Board. 
2/ Les techniques de calcul qui permettent de transformer et d'interpréter ces données expérimentales brutes avant de les appliquer au calcul de dose. Ce sont elles qui ont été plus particulièrement étudiées dans ce travail.

\subsection{Interprétation des données in vitro et in vivo}

Rappelons que la CIPR dans son nouveau modèle respiratoire décrit dans la publication 66 (CIPR, 1994), définit des types d'absorption $F, M$ et $S$ par des taux $s_{\mathrm{r}}, s_{\mathrm{S}}$ et $s_{\mathrm{b}}$ et des fractions $f_{\mathrm{r}}$ et $f_{\mathrm{b}}$ de transfert du poumon au sang. Il est admis que ces phénomènes d'absorption ou de transfert du poumon au sang sont indépendants des espèces animales, mais que par contre l'épuration mécanique est elle dépendante des espèces. À partir de ce postulat particulièrement bien décrit dans une étude européenne récente pour des particules de Cobalt (Kreyling et al., 1991), le NRPB a développé le logiciel GIGAFIT [Birchall, com. personnelle] d'interprétation des données in vivo (inhalation, instillation ou injection). Ce logiciel prend en compte les données d'épuration mécanique chez le rat (données provenant d'expérimentations réalisées à l'aide de particules inertes marquées (Kreyling, 1990)). À partir des résultats de rétention pulmonaire et sanguine, GIGAFIT permet par ajustement non linéaire de soustraire cette épuration mécanique et ainsi de calculer les taux $s_{\mathrm{r}}$, $s_{\mathrm{s}}$ et $s_{\mathrm{b}}$ et les fractions $f_{\mathrm{r}}$ et $f_{\mathrm{b}}$. Il faut donc, pour pratiquer ce calcul, disposer des données de rétention pulmonaire (exprimées en pourcentage à un temps $t$ de l'élément présent dans le poumon par rapport au dépôt initial pulmonaire) et des données de transfert au sang (qui correspondent dans le cas de l'uranium à la somme des pourcentages mesurés dans les organes cibles à un temps $t$, à savoir l'os, les reins et l'urine). Rappelons que dans des études antérieures (Ansoborlo et al., 1995a; Stradling et al., 1988), ces taux de transfert étaient évalués de façon analogue mais nettement simplifiée, en utilisant la formule suivante:

$$
F=0,693 / t(z / y)
$$

où $t$ est la période de décroissance pulmonaire, $z$ le pourcentage du dépôt initial passant dans le sang et y le pourcentage éliminé du poumon.

Pour ce qui concerne les données de dissolution in vitro l'exploitation des résultats est simple puisqu'il s'agit d'appliquer un ajustement par régression non linéaire (inclus également dans GIGAFIT) sous forme d'une somme de 2 exponentielles :

$\% \mathrm{U}$ cum. non dissous $=f_{\mathrm{r}} \exp \left(-0,693 t / T_{\mathrm{r}}\right)+\left(100-f_{\mathrm{r}}\right) \exp \left(-0,693 t / T_{\mathrm{s}}\right)$

où $f_{\mathrm{r}}$ est la fraction rapidement dissoute, $0,693 / T_{\mathrm{r}}=s_{\mathrm{r}}$ et $0,693 / T_{\mathrm{s}}=s_{\mathrm{s}}$ sont respectivement les taux rapidement et lentement dissous. Le but des tests in vitro est ainsi de disposer d'une approche intermédiaire relativement rapide et simple (tests conduits sur 15 à 30 jours) pour estimer la dissolution du composé, se situant entre les valeurs par défaut préconisées par la Publication 66 de la CIPR (ICRP, 1994), soit les types $F, M$ et $S$ et les valeurs spécifiques résultant de l'expérimentation in vivo. 
Ce rapport montre en particulier l'efficacité d'un tel logiciel pour l'interprétation indirecte de données expérimentales comme la surface spécifique ou l'hydratation, dont l'influence peut être estimée et surtout quantifiée par l'intermédiaire du calcul des fractions et taux de transfert au sang résultant d'essais in vitro ou in vivo.

\subsection{Calcul de la DPUI}

Le logiciel LUDEP qui intègre le nouveau modèle respiratoire de la CIPR 66 (ICRP, 1994), a été développé par le NRPB et permet d'effectuer les calculs de DPUI en utilisant soit les paramètres par défaut (DAMA de 1 ou $5 \mu \mathrm{m}$, débit ventilation de $1,2 \mathrm{~m}^{3} \mathrm{~h}^{-1}$, paramètres d'absorption types $F, M, S \ldots$ ), soit des paramètres spécifiques déterminés directement (DAMA, $\sigma_{\mathrm{g}}$ ), ou après interprétation des données expérimentales (les taux $s_{\mathrm{r}}$, $s_{\mathrm{s}}$ et $s_{\mathrm{b}}$ et fractions $f_{\mathrm{r}}$ et $f_{\mathrm{b}}$ ).

L'organigramme de ce logiciel dans l'ordre logique d'initiation des données s'appuie sur les fonctions suivantes:

a/ définition du régime d'incorporation (type d'exposition et de travailleur, régime chronique ou aigu, mode de contamination par inhalation, ingestion ou injection),

b/ identification du type de dépôt (DAMA, débit respiratoire, type de distribution...),

c/ évaluation du transport mécanique des particules (modèle de la Publication 66 (ICPR, 1994)),

$\mathrm{d} /$ entrée des fractions et taux d'absorption dans le sang (paramètres $s_{\mathrm{r}}, s_{\mathrm{s}}$ et $s_{\mathrm{b}}$ et $f_{\mathrm{r}}$ et $f_{\mathrm{b}}$ ) soit par défaut (types $F, M, S$ ) soit déterminés expérimentalement,

e/ sélection des radioisotopes et de leurs descendants (Publication 38 (ICRP, 1983)),

f/ choix des modèles biocinétiques : actuellement le logiciel ne propose que les modèles de la Publication 30 (ICRP, 1979) et de la Publication 54 (ICRP, 1988) avec la possibilité de rentrer ses propres données ou d'autres modèles existants. Cette partie du logiciel est en cours de développement,

g/ calcul de DPUI (corps entier, arbre respiratoire ou organes),

$\mathrm{h}$ / calcul des courbes de rétention ou d'excrétion en fonction des modèles biocinétiques cités précédemment.

Nous considérerons dans cet article le cas de la contamination aiguë par inhalation, représentative d'un incident au poste de travail.

\section{Résultats et discussion}

Afin d'illustrer de façon concrète le mode d'utilisation des données décrit dans le paragraphe matériel et méthode, nous présentons les résultats obtenus au cours d'une étude complète de spéciation sur le composé industriel $\mathrm{UO}_{2}$. Ce composé 
$\mathrm{UO}_{2}$, utilisé ensuite dans la fabrication des combustibles MOX ou mélange $(\mathrm{U}, \mathrm{Pu}) \mathrm{O}_{2}$, a été obtenu successivement par précipitation de nitrate d'uranyle appauvri $\mathrm{UO}_{2}\left(\mathrm{NO}_{3}\right)_{2}$ en diuranate d'ammonium (ADU) ou $\mathrm{U}_{2} \mathrm{O}_{7}\left(\mathrm{NH}_{4}\right)_{2}$, suivie d'une calcination en $\mathrm{U}_{3} \mathrm{O}_{8}$ et d'une réduction en $\mathrm{UO}_{2}$.

Les résultats sont présentés et interprétés dans l'ordre logique d'obtention des données. Les données mesurées directement (paramètres physico-chimiques) conduisent à un premier calcul de DPUI, et l'acquisition des données in vitro ou in vivo, qui nécessitent un traitement mathématique préalable, permet ensuite d'obtenir un calcul de plus en plus affiné de la DPUI.

1/ Les paramètres physico-chimiques mesurés directement et donc utilisables tels quels sans traitement des données, sont présentés dans le tableau I et correspondent à ce que l'on pourrait appeler une fiche d'identification du composé. L'ensemble de ces résultats et les paramètres de transfert au sang par défaut permettent d'effectuer un premier calcul de DPUI. En fonction des études déjà réalisées précédemment (Ansoborlo et al., 1989; Ansoborlo et al., 1995a ; Eidson, 1994; Métivier et al., 1992; Schieferdecker et al., 1985; Stradling et al., 1988), ce composé $\mathrm{UO}_{2}$ serait identifié de type $\mathrm{S}$. Les valeurs de DPUI ainsi obtenues avec le logiciel LUDEP, sont données dans la dernière ligne du tableau I. Le DAMA mesuré de 6,5 $\mu$ m (prélèvement effectué en sortie de four de réduction de l' $_{3} \mathrm{O}_{8}$ en $\mathrm{UO}_{2}$ ) étant supérieur à la valeur guide de $5 \mu \mathrm{m}$, la DPUI spécifique correspondante $\left(4,93 \times 10^{-6} \mathrm{~Sv} \mathrm{~Bq}{ }^{-1}\right)$ est inférieure à la valeur par défaut du Journal Officiel des Communautés Européennes (Journal Officiel, 1996) pour l'isotopie considérée $\left(5,87 \times 10^{-6} \mathrm{~Sv} \mathrm{~Bq}{ }^{-1}\right)$.

\section{TABLEAU I}

Paramètres physico-chimiques du composé $\mathrm{UO}_{2}$ et calcul de DPUI.

Physico-chemical parameters and calculation of DPUI for the $\mathrm{UO}_{2}$ compound.

\begin{tabular}{|c|c|c|}
\hline 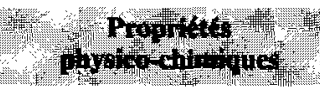 & $\begin{array}{l}\text { Parades par acfout } \\
\text { (CIPS 66) }\end{array}$ & 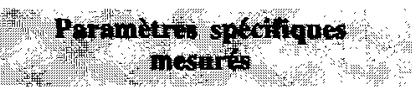 \\
\hline Densité & $3 \mathrm{~g} \mathrm{~cm}^{-3}$ & $9 \mathrm{~g} \mathrm{~cm}^{-3}$ \\
\hline Surface spécifique & non défini & $2,68 \mathrm{~m}^{2} \mathrm{~g}^{-1}$ \\
\hline $\begin{array}{l}\text { Spectrométrie de masse } \\
\text { Activité spécifique }\end{array}$ & choix libre de l'isotope & 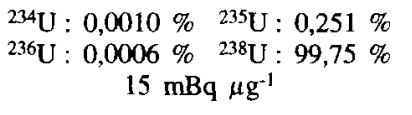 \\
\hline DAMA & $5 \mu \mathrm{m}$ & $6,5 \mu \mathrm{m}$ \\
\hline$\sigma_{\mathrm{g}}$ & 2,5 & 2 \\
\hline Système cristallin & non défini & $\mathrm{UO}_{2}$ \\
\hline DPUI $\left(\mathbf{S v} \mathbf{B q}^{-1}\right)$ * & $5,87 \times 10^{-6}$ & $4,93 \times 10^{-6}$ \\
\hline
\end{tabular}

* Ce calcul a été effectué en tenant compte de la composition isotopique de l'uranium appauvri et correspondant a $83,2 \% \mathrm{~d}^{238} \mathrm{U}, 15,5 \% \mathrm{~d}^{234} \mathrm{U}$ et $1,3 \%$ d ${ }^{235} \mathrm{U}$ en activité. 
2/ Les premiers résultats obtenus à 30 jours, en terme de dissolution et résultant directement de l'expérimentation in vitro sont donnés dans le tableau II.

\section{TABLEAU II}

Cinétique de dissolution in vitro de l'UO $\mathrm{O}_{2}$, exprimée en \% d'uranium cumulé dissous.

In vitro dissolution kinetic for $\mathrm{UO}_{2}$, expressed as \% of cumulated dissolved uranium.

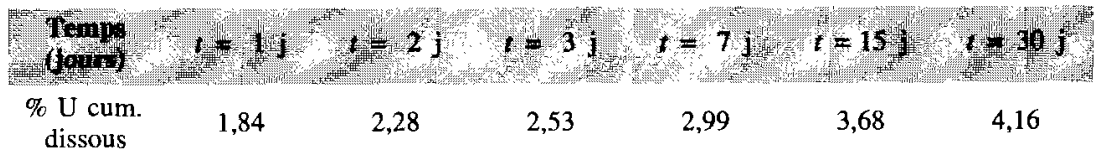

L'analyse de ces données permet d'obtenir les fractions dissoutes ainsi que les périodes de demi-dissolution, à savoir $f_{\mathrm{r}}=0,025, T=0,6 \mathrm{j}$ et $T_{\mathrm{s}}=1155 \mathrm{j}$. Les paramètres de transfert correspondants : $f_{\mathrm{r}}=0,025, s_{\mathrm{r}}=1,14 \mathrm{j}^{-1}$ et $s_{\mathrm{s}}=6,0 \times 10^{-4} \mathrm{j}^{-1}$, sont dérivés et conduisent à une DPUI de $3,65 \times 10^{-6} \mathrm{~Sv} \mathrm{~Bq}^{-1}$, en prenant pour le DAMA, la valeur mesurée en atelier de $6,5 \mu \mathrm{m}$.

La comparaison de ces résultats, avec ceux donnés dans la littérature, est difficile. En effet les composés $\mathrm{UO}_{2}$ utilisés ne sont pas toujours purs (traces d' $\mathrm{U}_{3} \mathrm{O}_{8}$, ou présences d'additifs industriels) et les paramètres physico-chimiques sont souvent incomplets (surface spécifique rarement citée). D'autre part les origines du composé sont très diverses en fonction du procédé industriel de fabrication (frittage, température d'obtention, voies humides ou sèches...), et enfin les tests utilisés et les milieux de dissolution très variés. Néanmoins, l'analyse globale des résultats trouvés chez certains auteurs (Ansoborlo, 1995a ; Eidson, 1994) montre que les composés $\mathrm{UO}_{2}$ étudiés sont très peu solubles (classe $Y$ de la Publication 30 (ICRP, 1979) et type $S$ de la Publication 66 (ICRP, 1994)), et qu'en moyenne le pourcentage dissous à $30 \mathrm{j}$ est compris entre 0,5 et $4 \%$. Le résultat de $4,2 \%$ que nous avons obtenu à $30 \mathrm{j}$ est donc très proche de ces valeurs, ce qui classerait cet $\mathrm{UO}_{2}$ en classe $\mathrm{Y}$ de la Publication 30 (ICRP, 1979) et entre les types $M$ et $S$ de la Publication 66 (ICRP, 1994). Cette comparaison met en évidence l'importance de la connaissance précise des caractéristiques physico-chimiques du composé.

3/ Les résultats de l'expérimentation in vivo sur rats, réalisée par dépôt intratrachéal de $480 \mu \mathrm{g}$ d'UO ${ }_{2}$, sont donnés dans le tableau III. Les pourcentages sont donnés par rapport au dépôt initial pulmonaire d'activité retenue au niveau des organes cibles (à savoir : le poumon, le foie, les reins, la carcasse) et d'activité éliminée dans les urines. 
Deux valeurs tests, découlant du tableau III, permettent de comparer ces résultats expérimentaux avec ceux trouvés dans la littérature. Le rapport $R /(R+U)=0,30$ à $t=1 \mathrm{j}, R$ correspondant au pourcentage d'uranium présent dans les reins et $U$ étant le pourcentage d'uranium présent dans les urines. Cette valeur est comprise dans l'intervalle de 0,3 à 0,4 défini par ailleurs (Stradling et al., 1988; Pasquier et Bourguignon, 1979). L'autre valeur test est le rapport $U / S=0,40$ à $t=1 \mathrm{j}, S$ étant le pourcentage d'uranium transféré au sang. Elle se trouve dans l'intervalle de 0,4 à 0,6 donné par Bertelli (Bertelli et Wrenn, 1994).

\section{TABLEAU III}

Résultats de l'expérimentation in vivo sur rats par dépôt intratrachéal d'UO $\mathrm{O}_{2}$ appauvri, exprimés pour chaque organe en pourcentage du dépôt initial pulmonaire.

Results of in vivo intratracheal deposit in rats on depleted $\mathrm{UO}_{2}$, expressed for each organ as fraction of initial lung deposit.

\begin{tabular}{|c|c|c|c|c|c|c|}
\hline (jows) & Powirons & Foe & Meins & Cancises & urro & $s^{4} x^{6}$ \\
\hline 1 & $61,5 \pm 10,3$ & $0,01 \pm 0,01$ & $0,32 \pm 0,16$ & $0,77 \pm 0,33$ & $0,74 \pm 0,03$ & $1,83 \pm 0,52$ \\
\hline 3 & $55,5 \pm 16,0$ & $0,01 \pm 0,01$ & $0,23 \pm 0,14$ & $0,81 \pm 0,57$ & $0,75 \pm 0,31$ & $1,79 \pm 0,87$ \\
\hline 7 & $55,1 \pm 6,4$ & $0,11 \pm 0,17$ & $0,18 \pm 0,04$ & $0,96 \pm 0,26$ & $1,51 \pm 0,24$ & $2,76 \pm 0,60$ \\
\hline 15 & $54,5 \pm 6,1$ & $0,32 \pm 0,17$ & $0,23 \pm 0,11$ & $1,01 \pm 0,41$ & $1,85 \pm 0,15$ & $3,41 \pm 0,21$ \\
\hline 30 & $53,5 \pm 3,8$ & $0,01 \pm 0,01$ & $0,27 \pm 0,13$ & $1,42 \pm 0,57$ & $2,19 \pm 0,53$ & $3,90 \pm 0,60$ \\
\hline 45 & $52,4 \pm 7,4$ & $0,04 \pm 0,04$ & $0,30 \pm 0,01$ & $4,93 \pm 1,71$ & $2,43 \pm 0,44$ & $7,71 \pm 2,11$ \\
\hline 60 & $51,5 \pm 6,3$ & $0,08 \pm 0,09$ & $0,38 \pm 0,12$ & $5,61 \pm 2,33$ & $2,67 \pm 0,05$ & $8,75 \pm 2,38^{c}$ \\
\hline 90 & $49,6 \pm 3,6$ & $0,66 \pm 0,72$ & $0,51 \pm 0,22$ & $5,64 \pm 2,13$ & $2,36 \pm 0,44$ & $9,17 \pm 1,75^{\mathrm{c}}$ \\
\hline
\end{tabular}

a : Moyenne \pm écart type sur 3 animaux, Dépôt Initial Pulmonaire $=479 \pm 85 \mu \mathrm{g} \mathrm{U}$ (appauvri ${ }^{235} \mathrm{U}$ ). b : Reins + carcasse + urine.

c : Valeurs estimées à partir du taux de transfert au sang $F=0,693 / T(z / y)(z \%$ sang, $y \%$ poumon), soit $44,4 \%$ rapidement dissous $\left(t_{1 / 2}=0,4 \mathrm{j}\right)$ pour $t<3 \mathrm{j}$ avec $F=0,089 \mathrm{j}^{-1}$ et $55,6 \%$ lentement dissous $\left(t_{1 / 2}=541 \mathrm{j}\right)$ soit, pour $t>3 \mathrm{j}, F=0,0016 \mathrm{j}^{-1}$.

L'analyse de ces données calculées manuellement conduit aux valeurs suivantes des paramètres spécifiques : $f_{\mathrm{r}}=0,444, s_{\mathrm{r}}=0,089 \mathrm{j}^{-1}$ et $s_{\mathrm{S}}=1,6 \times 10^{-3} \mathrm{j}^{-1}$ et à une DPUI de $1,96 \times 10^{-6} \mathrm{~Sv} \mathrm{~Bq}^{-1}$, en prenant pour le DAMA, la valeur mesurée en atelier de $6,5 \mu \mathrm{m}$.

Les mêmes données traitées à l'aide du logiciel GIGAFTT, à partir de l'ajustement non linéaire des cinétiques de décroissance pulmonaire et de la charge sanguine, donnent les valeurs suivantes pour les paramètres de transfert sanguin : $f_{\mathrm{r}}=0,133, s_{\mathrm{r}}=1,62 \mathrm{j}^{-1}, s_{\mathrm{s}}=5,410^{-3} \mathrm{j}^{-1}, f_{\mathrm{b}}=0,753$ et $s_{\mathrm{b}}=1,46 \times 10^{-3} \mathrm{j}^{-1}$, et la DPUI 
de $2,84 \times 10^{-6} \mathrm{~Sv} \mathrm{~Bq}^{-1}$, en prenant pour le DAMA, la valeur mesurée en atelier de $6,5 \mu \mathrm{m}$.

4/ L'ensemble des valeurs des paramètres de transfert ainsi que les DPUI calculées ont été regroupées dans le tableau IV. On notera que les paramètres physiques spécifiques ne suffisent pas à eux seuls à évaluer «correctement » la DPUI ( $3^{\mathrm{e}}$ colonne). Le principal commentaire résultant de cette comparaison est qu'entre la valeur par défaut de la DPUI pour un composé de type $S$ ( $2^{\mathrm{e}}$ colonne) et la valeur in vivo obtenue en utilisant GIGAFIT, une diminution d'un facteur 2 est observée (dernière colonne). Exprimé en terme de Limite Annuelle d'Incorporation ou LAI, ce résultat correspondrait à une augmentation de $3410 \mathrm{~Bq}$ (valeur par défaut pour un type $S$ ), à $7040 \mathrm{~Bq}$ de la LAI spécifique.

\section{TABLEAU IV}

Tableau récapitulatif des différentes DPUI calculées en partant des valeurs par défaut de la Publication 66 et en fonction des paramètres spécifiques déterminés expérimentalement.

Summary of different DPUI calculated either from default values of Publication 66, or from specific parameters resulting from experimental assessment.

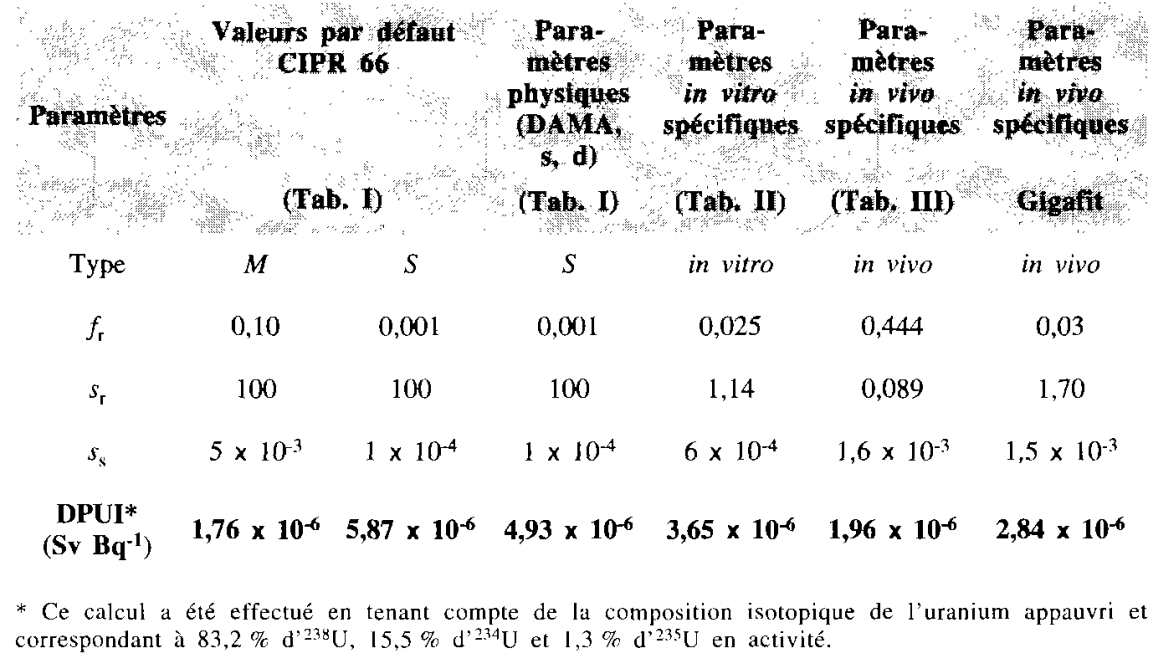

En parallèle au traitement de ces données, il est intéressant de confronter les résultats obtenus in vivo, aux résultats in vitro et à ceux décrits dans la littérature.

En ce qui concerne les résultats in vitro les pourcentages dissous à $t=1 \mathrm{j}$ $(1,84 \%)$ et $t=30 \mathrm{j}(4,16 \%)$ en milieu de culture cellulaire sont très bien 
corrélés à ceux, obtenus in vivo, pour le pourcentage transféré au sang qui sont respectivement de $1,83 \%$ à $t=1 \mathrm{j}$ et $3,90 \%$ à $t=30 \mathrm{j}$. Il semble donc dans le cas précis de ce composé $\mathrm{UO}_{2}$ que le milieu de culture cellulaire soit le milieu représentatif de la dissolution et du transfert au sang.

La comparaison des résultats in vivo, comme précédemment pour les essais in vitro, avec ceux donnés dans la littérature est difficile à réaliser. Par exemple la surface spécifique qui joue un rôle essentiel dans les phénomènes de dissolution, n'est que trop rarement citée. De plus les interprétations sont très variables entre les auteurs, ce qui explique le choix fait dans cette étude de présenter les résultats sous différentes formes: périodes de décroissance pulmonaire, pourcentage de transfert au sang et enfin calcul des fractions et taux de transfert sanguin définis dans la Publication 66 (ICRP, 1994).

Si l'on fait une synthèse des principaux résultats publiés (Ansoborlo et al., 1995a ; Eidson, 1994 ; Métivier et al., 1992 ; Schieferdecker et al., 1985 ; Stradling et al., 1988), on constate que les périodes lentes de décroissance pulmonaire varient suivant les espèces et les individus : 170 à $330 \mathrm{j}$ chez le rat, 350 à $450 \mathrm{j}$ chez le babouin, 180 à $480 \mathrm{j}$ chez le chien et de 110 à $1900 \mathrm{j}$ chez l'homme. La période de décroissance pulmonaire lente mesurée chez le rat dans la présente étude est de $541 \mathrm{j}$. Cette valeur est sensiblement supérieure à l'intervalle des valeurs précitées chez le rat ce qui n'est pas surprenant étant donné les grandes variations inter-souches chez cette espèce. Une étude effectuée par Stradling (Stradling et al., 1988) chez le rat par inhalation et instillation, et concernant 2 composés $\mathrm{UO}_{2}$ (fritté et non fritté), fournit des résultats exprimés sous forme de pourcentage transféré au sang très comparables : 1,6 à $3,5 \%$ à $t=7 \mathrm{j}$ et 2,3 à $6,8 \%$ à $t=84 \mathrm{j}$, comparés respectivement à $2,76 \%$ à $t=7 \mathrm{j}$ et $9 \%$ à $t=90 \mathrm{j}$ dans cette étude. Dans les deux cas, le composé $\mathrm{UO}_{2}$ se situe entre les types $M$ et $S$ de la Publication 66 (ICRP, 1994).

L'ensemble de ces remarques, et plus particulièrement la grande dispersion des résultats observés in vivo selon différents auteurs, souligne l'importance d'une caractérisation précise tant sur le plan physico-chimique que biologique du composé étudié, et le problème de l'uniformité des modes d'interprétation.

Enfin et pour démontrer l'intérêt pratique de l'exploitation des données spécifiques expérimentales, nous avons utilisé la fonction offerte par LUDEP de simuler les fonctions d'excrétion urinaire ou de décroissance pulmonaire correspondantes. Ces résultats sont présentés à la figure 1. Ce type de courbes trouve son intérêt dans le cadre de la surveillance médicale des travailleurs susceptibles d'être exposés. 
INTERPRÉTATION DE DONNÉES POUR LE CACUL DE DOSE

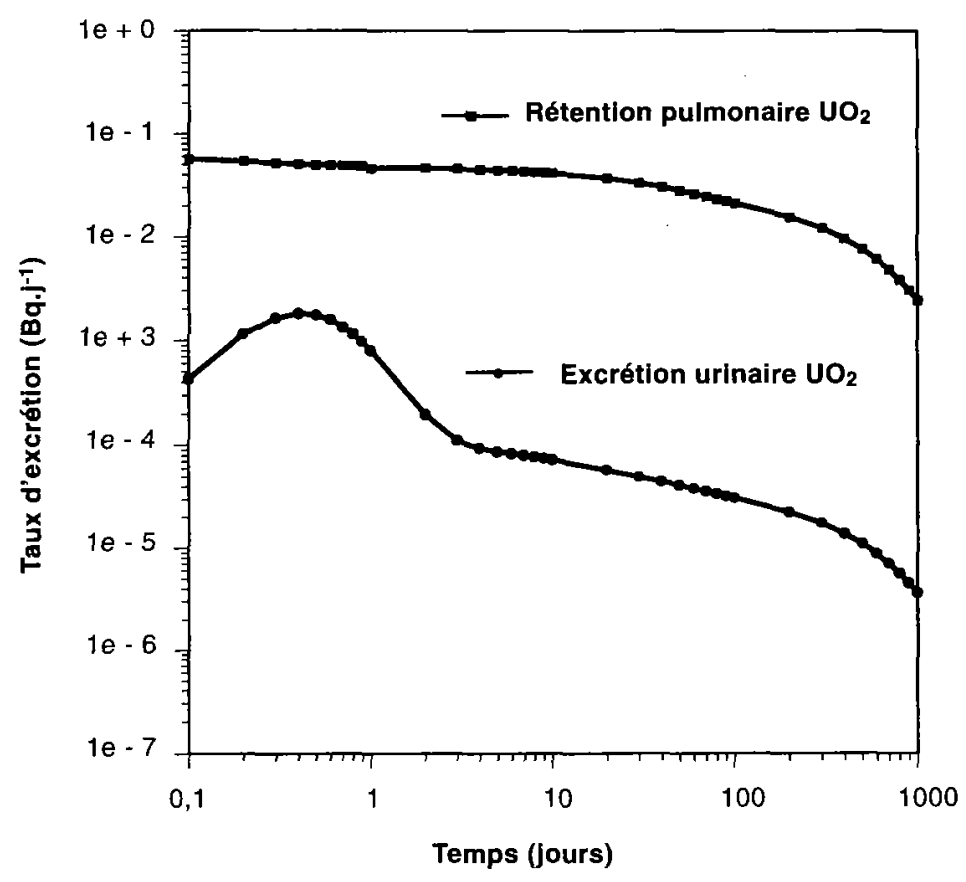

Fig. 1. - Courbes prédictives de la décroissance pulmonaire et de l'excrétion urinaire chez le travailleur standard après contamination aiguë $(\mathrm{I} B q)$ par inhalation d' $U \mathrm{O}_{2}$ appauvri, pour un DAMA de 6,5 um et des paramètres spécifiques déterminés expérimentalement.

Predicted curves from pulmonary decrease or urinary excretion (reference values for workers) after acute contamination (I Bq) of depleted $\mathrm{UO}_{2}$, for an $\mathrm{AMAD}$ of $6,5 \mu \mathrm{m}$, and specific parameters resulting from experimentation.

\section{Conclusion}

La Commission Internationale de Protection Radiologique ainsi que le Journal Officiel des communautés européennes, dans leurs recommandations récentes, donnent des valeurs guides par défaut pour les paramètres physico-chimiques et biologiques. Ces valeurs conduisent à des DPUI de référence pour chaque isotope d'un élément donné en fonction de son type $(F, M, S)$ de transfert. Le nouveau modèle respiratoire de la Publication 66 (ICRP, 1994) présente par 
ailleurs une grande flexibilité, qui se traduit par la possibilité d'utiliser, lorsqu'ils sont disponibles, des paramètres spécifiques déterminés expérimentalement. Le but de cette étude a été de présenter les méthodes successivement utilisées pour déterminer ces paramètres (spéciation physicochimique, tests in vitro et tests in vivo) et de montrer qu'au fur et à mesure de cette évaluation le calcul de la DPUI s'affinait, ceci en utilisant des outils d'interprétation des données de plus en plus performants (logiciels GIGAFIT et LUDEP). Cette méthodologie a été appliquée au composé industriel $\mathrm{UO}_{2}$ (uranium appauvri), utilisé dans la fabrication du combustible MOX. Ce composé bien défini au point de vue physico-chimique (DAMA $=6,5 \mu \mathrm{m}$, Surface spécifique $=2,68 \mathrm{~m}^{2} \mathrm{~g}^{-1}$ ), a été testé in vitro et in vivo (par dépôt intratrachéal), ce qui a permis de calculer les fractions et taux de transfert, respectivement $f_{\mathrm{r}}$, $s_{\mathrm{r}}$ et $s_{\mathrm{s}}$ et ainsi que les DPUI correspondantes, dont principalement une DPUI spécifique égale à $2,84 \times 10^{-6} \mathrm{~Sv} \mathrm{~Bq}{ }^{-1}$. Ce composé $\mathrm{UO}_{2}$ est ainsi classé comme un composé de classe Y suivant la Publication 30 (ICRP, 1979) et entre les types $M$ et $S$ de la Publication 66 (ICRP, 1994). L'ensemble de ces résultats souligne l'importance d'une connaissance précise d'une part de tous les paramètres physico-chimiques et biologiques et d'autre part des techniques d'interprétation de ces données qui sont en pleine évolution. Cette étude montre donc l'intérêt d'une analyse des données en terme d'excrétion et de rétention dans divers organes sur la base des paramètres spécifiques du composé industriel. Enfin remarquons l'utilité de ces outils qui permettent d'intégrer les paramètres spécifiques des composés pour évaluer les courbes d'excrétion et de rétention dans divers organes, ce qui peut être particulièrement utile pour l'interprétation des résultats de la surveillance médicale des travailleurs de l'industrie de l'uranium.

Remerciements. Les auteurs remercient tout particulièrement les techniciens du SDOS/LEAR qui ont participé à ce travail ainsi que N. Stradling, A. Hodgson et A. Birchall du NRPB pour leurs conseils dans l'utilisation de GIGAFIT.

\section{RÉFÉRENCES}

Ansoborlo E., Bérard P., Chalabreysse J. (1989) Study of industrial exposure to class Y uranium compounds: methods and results. Radiat. Prot. Dosim., 26, 101-105.

Ansoborlo E., Hengé-Napoli M.H., Rannou A., Pihet P., Dewez P. (1995a) French experience in the field of internal dosimetry assessment at a nuclear workplace: methods and result on industrial uranium dioxide. J. Radioanal. Nucl. Chem., 197, 161-172.

Ansoborlo E., Hengé-Napoli M.H., Roy M., Pihet P., Rannou A. (1995b) Implementation of the new ICRP respiratory tract model : Example of a potential exposure to an industrial oxide $\mathrm{UO}_{2}$. Bull. Radiat. Prot., I8, 1-7.

Bertelli L., Wrenn M.E. (1994) Interpretation of in vivo and bioassay results and dose and ALI calculations using a new uranium model. Radiat. Prot. Dosim., 53, 277-280. 
Birchall A., Bailey M.R., James A.C. (1991) LUDEP : A lung dose evaluation program. Radiat. Prot. Dosim., 38, 167-174.

Cooke H., Holt F.B. (1974) The solubility of some uranium compounds in simulated lung fluid. Health Phys., 4, 27, 69-77.

Eidson A.F. (1994) The effect of solubility on inhaled uranium compound clearance : a review. Health Phys., 67, 1-14.

ICRP (1979) Limits for intakes of radionuclides by workers, ICRP publication 30, Pergamon press, Oxford.

ICRP (1983) Radionuclide transformations : energy and intensity of emissions, ICRP Publication 38. Pergamon press, Oxford.

ICRP (1988) Individual monitoring for intakes of radionuclides by workers: design and interpretation, ICRP Publication 54. Pergamon press, Oxford.

ICRP (1990) Recommendations of the International Commission on Radiological Protection, ICRP publication 60. Pergamon press, Oxford.

ICRP (1994) Human respiratory tract model for radiological protection, ICRP publication 66. Pergamon press, Oxford.

Jarvis N.S., Birchall A. (1994) LUDEP 1.0, un logiciel pour ordinateur personnel qui applique le nouveau modèle pulmonaire de la CIPR. Radioprotection, 29, (1), 81-86.

Journal Officiel Des Communautés Européennes. (1996), ISSN 0378-7060, L 159, 29/06/96.

Kalkwarf D.R. (1983) Dissolution rates of uranium compounds in simulated lung fluids. The Science of the total environment, 28, 405-414.

Kreyling W.G. (1990) Interspecies comparison of lung clearance of insoluble particles. J. Aerosol. Medicine, 93-110.

Kreyling W.G., André S., Collier C.G., Ferron G.A., Métivier H., Schumann G. (1991) Interspecies comparison of lung clearance after inhalation of monodisperse, solid cobalt oxide aerosol particle. J. Aerosol. Sci., 509-535.

Métivier H., Poncy J.L., Rateau G., Stradling G.N. (1992) Uranium behaviour in the baboon after the deposition of a ceramic form of uranium dioxide and octoxide in the lungs: implications for human exposure. Radioprotection, 27, 263-281.

Pasquier C., Bourguignon M. (1979) Etude expérimentale de la fixation rénale après contamination aigue par l'uranium - Influence de la solubilité des composés utilisés : $\mathrm{UO}_{2}^{2+}, \mathrm{UO}_{3}, \mathrm{U}_{3} \mathrm{O}_{8}$. IAEASM-237/29.

Schieferdecker H, Dilger H., Doerfel H. (1985) Inhalation of uranium aerosols from $\mathrm{UO}_{2}$ fuel element fabrication. Health Phys., 48, 29-48.

Stradling G.N., Stather J.W., Gray S.A. (1988) The metabolism of ceramic and non ceramic forms of $\mathrm{UO}_{2}$ after deposition in the rat lung. Human. Toxicol., 7, 133-139.

Surya Narayana D.S., Sundararajan A.R., Harvey J. (1994) Characterization of uranium oxide aerosols. J. Aerosol. Sci., 25, 909-922. 